\title{
Development, Evolution, and Assessment of a Momentum-Variable- Based Procedure for the Prediction of Flow at All Speeds
}

\author{
G.E. Schneider \\ University of Waterloo, Canada \\ 200 University Avenue West, Waterloo, ON N2L 3G1 \\ gerry.schneider@uwaterloo.ca
}

The development history of resolving the pressure-checkerboard problem for co-located variable discrete formulations will be reviewed. The original motivation that led to the physical influence scheme will be presented. From that initial, partially successful, effort at resolving the pressure-checkerboard problem, it remained that the Euler equations would permit very specific pressure oscillation 'solutions'. It will be reviewed how the examination of the Euler equations led to the development of an all-speed flow algorithm. The details and elements of an all-speed flow algorithm will be reviewed. Solution methods for the coupled system of equations will be discussed. After the formulation has been fully reviewed, several examples of application of the algorithm to incompressible flows, compressible flows, two-phase flows, and chemically reacting flows will be presented. 